\title{
ERRATUM 2
}

To the article "Synthesis of novel macrocyclic and heterocyclic taurine derivatives based on the reaction of sodium 2-[(4,4-diethoxybutyl)amino]ethanesulfonate with phenols"

by Andrey V. Smolobochkin, Lola Zh. Yakhshilikova, Almir S. Gazinov, Liliya I. Vagapova, Alexander R. Burilov, Michael A. Pudovik

Chemistry of Heterocyclic Compounds, Vol. 56, No. 7, pp. 888-891, July, 2020.

In the title articles and in the Table of content Gazinov should read Gazizov. 